\title{
An Overview of Integration of Mobile Infrastructure with SDN/NFV Networks
}

\author{
Ángel Leonardo Valdivieso Caraguay, Lorena Isabel Barona López, Luis Javier García Villalba \\ Group of Analysis, Security and Systems (GASS) \\ Department of Software Engineering and Artificial Intelligence (DISIA) \\ Faculty of Information Technology and Computer Science, Office 431 \\ Universidad Complutense de Madrid (UCM) \\ Calle Profesor José García Santesmases, 9 \\ Ciudad Universitaria, 28040 Madrid, Spain \\ Email: \{angevald, lorebaro\}@ucm.es, javiergv@fdi.ucm.es
}

\begin{abstract}
The growing number of on-line applications and services running on wireless and mobile devices has been limited by the rigidity of actual IT infrastructure, in which the closed union between data and control planes limits the possibility of customize the network behavior. In this context, the concepts of SDN and NFV appear as a viable solution to open the infrastructure to developers in order to create new services and applications. In this work, we describe the concepts of SDN, NFV and analyze the possibility of integrate these technologies in mobile networks. Furthermore, we present the last projects and an architecture proposal focused on this direction. Finally, we discuss the trends and challenges in order to implement these advances in production networks.
\end{abstract}

\section{Keywords—Mobile Network; Network Function Virtualization; OpenFlow; Software Defined Networking.}

\section{INTRODUCTION}

The diversity of network infrastructures has enabled the increase of connectivity among users and consequently it has promoted the establishment of new business models. This new digital environment requires an IT infrastructure capable to ensure high level of Quality of Service (QoS) and customization of applications. However, the heterogeneity of cellular and wireless technologies and the current configuration techniques complicate the control and management of the network.

The IT infrastructure is composed by a set of hardware devices running proprietary software that analyzes the traffic and selects the optimal route to the destination. In this scenario, the network administrator does not have access to modify the internal operation of the device. Instead, the administrator can only configure a minimum set of parameters to modify the network behavior. Moreover, the inclusion of new services requires the individual updating of devices or the complete replacement of hardware infrastructure. For this reasons, the idea of separate the data plane and control plane in order to customize the network behavior has gained importance. Similarly, the possibility of encapsulate the different network functions based on actual network conditions can optimize the allocation of available resources.

The concepts of Software Defined Networking (SDN) and Network Function Virtualization (NFV) have changed the vision of typical network infrastructure. SDN separates the data and control planes in network devices and establishes a centralized control of the network behavior. This architecture enables to the network administrator the possibility to design and develop "network applications" and dynamically control the network. For its part, NFV allows the deployment of virtualized network functions (e.g. load balancers, firewalls) as virtual instances over standardized hardware (storage, network and servers). This technology integrates the use of different resources (servers, storage, IT-hardware), enhances the scalability of the network services and reduces the capital and operational cost.

A techno-economic analysis in mobile infrastructure reveals that the benefits of the introduction of SDN and virtualization techniques could decrease the capital expenditures. The capital expenditure could be reduced around $13.81 \%$ in a SDN scenario [1]. It is clear that architectures based on SDN offer multiple potential advantages for telecom operators [2], for instance, the possibility of deploy Radio Base Stations in the Cloud [3] or integrating LTE network elements with SDN switches managed from the cloud [4]. In this context, the industry and research community go a step further in this direction and have been combining their efforts in multiple projects such as OpenRoads [5], SoftCell [6] as well as European Projects such as T-NOVA [7], UNIFY [8] among others. In this piece of work, we describe the concept and the evolution of SDN and NFV in the last years. Furthermore, the integration of mobile infrastructure with SDN/NFV as well as the trend and challenges to implement these technologies in production networks are analyzed.

The work is structured as follows: in Chapter II the concepts of SDN and NFV are presented. Next, Chapter III reviews the integration of mobile networks with both 
technologies. Chapter IV analyzes the trends and challenges and provides an initial SD/NFV architecture. Finally, Chapter $\mathrm{V}$ presents the conclusions.

\section{SDN/NFV}

A typical network device is composed by an integrated data plane and control plane. The data plane receives the packet, reads the header information, sends the information to the control plane and forwards the packet to the next network device. For its part, the control plane analyzes the information provided by the data plane and executes a routing algorithm to establish the optimal route to the destination. Once the route is chosen, the control plane sends the decision to the data plane. However, the limited coordination and access to the configuration of the devices (closed technology) has limited the development of customized network applications and QoS services.

Software Defined Networking is a new network paradigm that removes the rigidity present on current architectures and improves flexibility and management in networks. SDN decouples the control plane and the data plane in network devices and establish an open communication interface between them. In addition, SDN proposes a centralized control of the network and open APIs to facilitate the development of high level network applications and services. OpenFlow is the first SDN standard that has been widely used in different research projects [9] [10]. OpenFlow is designed based on the actual flow tables located in traditional network devices and opens those up. The controller uses the OpenFlow protocol [11] to configure the flow tables in switches. Figure 1 shows the differences between SDN and traditional architectures.

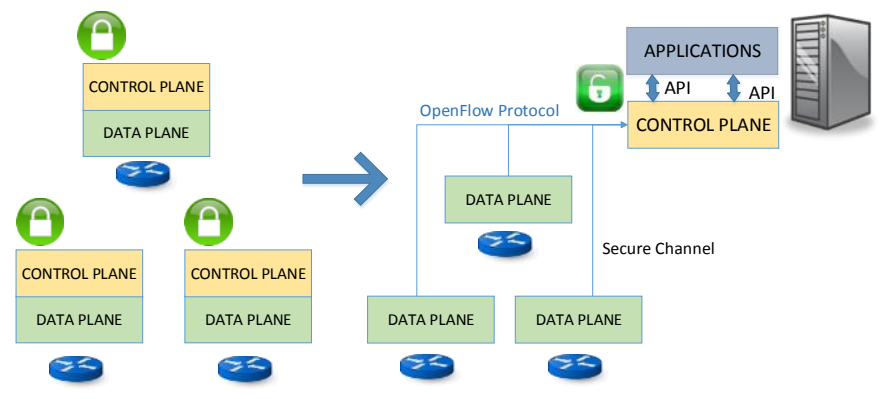

Fig. 1. Comparison between traditional and SDN architectures [10]

Another limitation of the actual infrastructure is the highly amount of network devices, each operating their own private software and highly dependent in proprietary hardware. For this reason, the design and installation of new services usually require the individual software updating or the replacement of hardware. This rigidity increases the installation and operational costs. In this context, the Network Function Virtualization NFV concept has gained in importance in the telecommunications industry.

Network Function Virtualization proposes the transferring of the different network functions (routing, firewall, deep packet inspection DPI, gateway) as virtual software-based applications executed in IT platforms (servers, switches and storage). This new vision of IT services provides a major flexibility and scalability, facilitates the development cycles and reduce costs. Figure 2 describes the differences between NFV and traditional architectures.

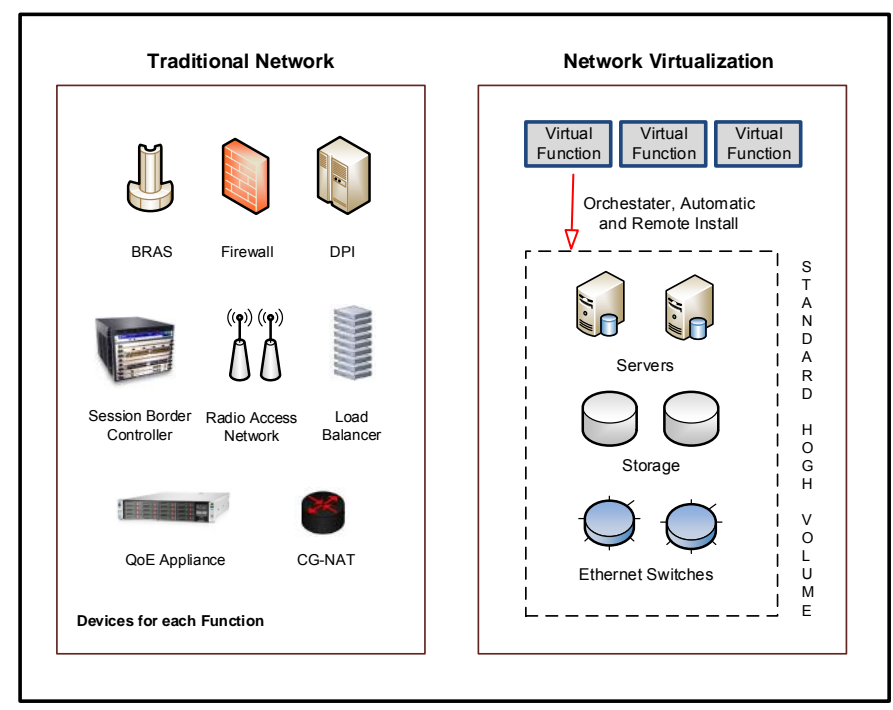

Fig. 2. Comparison between traditional and NFV paradigms

The NFV architecture identifies three principal modules: the Network Function Virtualization Infrastructure (NFVI) that includes all hardware resources, the Virtualized Network Function (VNF) that represents a network functions executed over the NFVI and the NFV Management and Orchestrator (NFV M\&O) responsible for coordinate the execution of the different network functions (NF) over the infrastructure [12].

It is important to note that SDN and NFV concepts are different but complementary. Thanks to the SDN separation of data and control planes in network devices, the integration of NFV in virtualized IT environments is feasible. However, the implementation in production networks has several challenges to be addressed [13].

\section{INTEGRATION OF WIRELESS NETWORKS WITH SDN/NFV}

In the last years, SDN approach has been expanded to mobile networks, giving rise the concept of Software Defined Wireless Networks [5] [14] [15] [16] or Wireless Mesh Software Defined Networks [17] [18]. Similarly, the integration between SDN and mobile technologies (LTE) has gained the attention of industry and research community [19] [20] [21] as well as the close relationship between SDN and NFV. For instance, EmPOWER [22] shows a testbed composed of 30 nodes that facilitates the deployment of SDN/NFV experiments for WIFI networks and it also provides monitoring tools in order to control the energy consumption. In the wireless field, some research intends to apply OpenFlow in order to enhance these types of networks. 
OpenRoads [5] presents an architecture of three layers (flow, slicing and controller) based on OpenFlow protocol and SNMP in order to innovate in WIMAX and WIFI networks. In the same way as OpenFlow wired networks, the wireless devices (Access Point or Wimax base station) have a flow table which are controlled through the controller. For its part, the slicing layer divides the data traffic through the FlowVisor tool [23] and NOX controller is the brain of the network control. The deployment contains 85 Access Points and two Wimax Base stations (over Stanford campus network) and provides functions such as hard handover, bicasting, Hoolock, among others.

Dely et al. in [18] presents an approach to improve the mobility in Wireless Mesh Networks (WMN). For this purpose, it introduces a mesh router known as a Mesh Access Point (MAP), which forwarding the traffic to the destination through other mesh routers or gateways. It is important to note that MAPs have OpenFlow support. Each node has some physical wireless cards and these in turn are divided into two virtual interfaces, one related with the data plane and the other for control traffic. The data interface is related with OpenFlow datapath. The gateways allow connectivity with the outside and each mesh router has an agent in order to monitoring the links, channel utilization, and others. Moreover the core network has two elements: the Monitoring and Control Server (MCS) and NOX controller. MCS builds a topology database with the information from mesh routers and NOX controls the mesh network. Proofs of concept were conducted over an experimental Wireless Broadband Mesh Network (KAUMesh), which is based on $802.11 \mathrm{a} / \mathrm{b} / \mathrm{g}$ standard. These tests were focused on mobility capacities, when clients move rapidly between different MAPs.

Regarding to cellular networks, the advances are limited and not homogeneous due each research applies different approaches and focuses on diverse elements, the Radio Access network (RAN) and the Evolved Packet Core (EPC).

On one hand, SoftRAN [24] framework uses the SDN concept in order to improve the RAN performance. SoftRAN has a whole view of interference and load of each node and in this way coordinates the allocation of radio resources, especially in dense networks. Each base station sends periodically information to controller and it is saved in a data base, which contains the following elements: an interference map, the flow records and the network operator preferences. SoftRAN was tested with some use cases such as load balancing and utility optimization.

On the other hand, there are some approaches focused on the core part of cellular networks. CellSDN [25] [26] provides an architecture with advances characteristics, such as the slicing of the network resources, better packet classification through deep packet inspection functionalities, scalability via local switch agents and the creation of applications based on the user attributes (network provider, device type).

For its parts, SoftCell [6] enhances the scalability and flexibility in SDN/LTE networks through the analysis of workload and the implementation of fine-grained policies.
Softcell also aggregates the traffic based on different aspects such as the base station, mobile devices and the service polices. Each base station is connected with an access switch. This switch has OpenFlow support and is supervised by the controller.

Similarly, MobileFlow [21] takes advantage from SDN and data center concepts to enable and foster the innovation in carrier networks. The main elements of the architecture are MobileFlow Forwarding Engine (MFFE) and MobileFlow Controller (MFC). MMFE has support to mobile network tunnel capacities and allows the integration with legacy EPC equipment. For this reason, the MMFE is considered the data plane. Each MMFE is controlled by the MFC (control plane). The implementation and validation process consists on a prototype based on $\mathrm{x} 86$ servers and OpenFlow components.

Furthermore, some projects could be applied to SDN/mobile networks (Wireless and cellular). These projects not only take into account SDN technology but also another key enabler technologies such as NFV, cloud computing, advances virtualization techniques, among others.

For instance, T-NOVA project [7] aims the design and implementation of a framework to allow operators the deployment of virtualized Network Functions (NF) over Network/IT infrastructures. This virtual network appliances are developed in software using SDN/NFV and eliminate the need of acquire, install and maintain specialized hardware. The framework will enable an open API for developers to the design and develop of NF appliances.

UNIFY (Unifying Cloud and Carrier Networks) [8] considers the entire network (home networks to data centers) as a "unified production environment", focusing on telco functions. UNIFY combines the benefits of cloud computing and virtualization in order to build a new architecture that optimizes data traffic flows and allows the dynamic placement of networking, computer and storage components. The consortium creates a model with advanced programmability, new languages, algorithms and management tools to optimize data traffic across networks. UNIFY intends to design a universal hardware node in order to support network functions and traditional data center workloads. The whole architecture allows agility (velocity), simplicity (automation), flexibility (granularity) and programmability of the services, providing and open environment for the deployment of these services and, at the same time, reducing the costs. UNIFY will derive a framework which supports a variety of services such as OpenFlow, Network Function Virtualization, and so on. Besides, this project is focused on three areas. First, infrastructure virtualization, second, flexible service chaining and thirdly, network service chain invocation (programmability interfaces).

CROWD project (Connectivity management for eneRgy Optimised Wireless Dense networks) [27] proposes a novel architecture in order to enhance very dense and heterogeneous wireless networks (Dense Nets). CROWD promotes a paradigm change in this kind of networks through global network cooperation, fine and dynamic network configuration, 
resources on demand, among others. For this purpose, this project uses SDN and OpenFlow protocol as an enabler concepts to control and manage in an efficient way the resources of Dense Nets. CROWD architecture has two kind of controllers: local and regional. The infrastructure layer consists of base stations (eNBs or Wifi AP) which are configurable via OpenFlow. CROWD provides dynamic controller placement, dynamic backhaul reconfiguration, energy optimization, MAC optimization mechanism and ensures user quality of experience.

CITYFLOW project (OpenFlow City Experiment Linking Infrastructure and Applications) [28] introduces the use of virtual path slice (VPS) technology at large scale on an OpenFlow network. This project emulates a city with one million inhabitants, with OpenFlow support and taking into account network topologies over xDSL, LTE and Fiber technologies.

Moreover, there are some facilities that allow the experimentation with SDN in wireless environment, such as OFELIA (Open Flow in Europe: Linking Infrastructure and Applications) [29] and the above mentioned deployments, OpenRoads [5] and KAUMesh [18]. OFELIA provides an environment to investigate and validate revolutionary ideas. OFELIA has a set on ten islands over Europe based on OpenFlow technology. Likewise, SmartFIRE [30] develops a large-scale testbed located in South Korea and Europe. The European zone is composed by three different and heterogeneous islands. Two islands belong to the OFELIA project (iMinds and UMU) and the other is part of the OpenLab federation testbed (UTH testbed). For its part, South Korea testbed includes OpenFlow islands in different institutes, for example Electronics and Telecommunications Research Institute (ETRI) and Seoul National University (SNU).

All these advancements are in an early stage but the initial results are promising. Next, we present the current trends and challenges and a possible architecture aligned with the SDN and NFV concepts.

\section{TRENDS AND CHALLENGES}

Nowadays, the variety of mobile networks providing different services and applications requires a mobile infrastructure capable of provide high levels of security, performance and QoS. This means that current mobile networks requires a standardized environment, wherein foster the innovation and introduction of new services would be possible in less time and with the lowest investment. This may be achieved through the synergy of NFV and SDN. On one hand, SDN enhances the control and management of network devices through the centralized control. On the other hand, NFV reduces the investment by means of sharing resources not only physical infrastructures but also network functions. This means the reduction in capital (Capex) and operational costs (Opex) that is the main limitation of carrier and service providers. In the context of mobile networks, there are some challenges that a
SDN/NFV approach may solve. Next, we describe the actual issues and trends.

Rapid innovation: The combination of SDN and NFV reduces the time to market of new services, through the resource virtualization and centralized control in different locations over an standardized environment. This eliminates the vendor dependence and increases the benefits for stakeholders.

Mobile traffic monitoring and management: SDN allows fine-grained control of the network data traffic and resources. This is especially important for handover, where OpenFlow may facilitate the change between nodes. Additionally, the traffic could be classified and managed based on the kind of flow, aggregation criteria (cell, user equipment, etc), flow rate, occupation of the resources (channels, links, base stations or AP, available bandwidth), among others. For instance, could be possible connect users to multiple networks or defines threshold parameters (bandwidth, location), allowing the easy change between them. Other applications may include the dynamic resources management of wireless backhaul or the capacity aggregation not only with one technology but also combining different technologies.

Energy efficiency: The traffic load is changing constantly according different factors, time, location, special events, among others. On one hand, SDN may enable the optimization of the power consumption based on real time conditions. In this way, the SDN controller could increase or decrease the number of resources allocated. On the other hand, NFV may decrease the number of devices due its flexibility and sharing capabilities.

Scalability and flexibility: Nowadays, the introduction or extension of new services is not easy because current architectures are closed. It process requires a long time or in some case is not performed due the investment is greater than the economy benefits SDN/NFV facilitates the service scalability and allows the reutilization of infrastructures and applications. Moreover, mobile networks are more flexible because SDN/NFV approach is aware of network conditions and changing traffic patterns.

Sharing infrastructure or services: SDN monitoring and VNF virtualization capabilities enable to share infrastructures and network resources. A service provider (SP) could deploy their network functions in the infrastructure of another SP, or use the applications (of another SP) in their own infrastructure. All of these activities are managed by the SDN controller. As a result of this, SDN/NFV introduces new capabilities in billing services. This generates more revenue for stakeholders, the first SP obtains revenue from the service and the second with the infrastructure lease. However, this is an ideal environment to network business; there are some legacy concerns that would be solved or agreed before this scheme can perform.

Inter-Cell Interference: Several APs or base stations in the same location could produce interference each other due the cell overlapping, bad coordination of subcarriers, among others. Consequently, it produces degradation of quality of services (QoS). In this context, SDN enables the easy 
management of radio resources by means the centralized control and the global view of the network.

Security: The full picture of network events of SDN allows a better control and the detection of anomalous activities. The controller could provide pieces of software that acts like Intrusion Detection System (IDS), firewalls or another security function.

The advances in these concerns are in preliminary state and require the effort and coordination of vendors, researchers and the organism working in these areas, such as Open Networking Foundation (ONF), International Telecommunication Union (ITU) or the European Telecommunications Standards Institute (ETSI), among others [31].

The Wireless and Mobile Working Group (WMWG) aids to promote and extend the ONF approaches in this field, it includes the incorporation of OpenFlow protocol with mobile networks, following the current standards such as 3GPP, IEEE and others. ITU tries to standardize SDN for telecom carriers. For instance, Joint Coordination Activity on SDN (JCA-SDN) coordinates the ideas from different Standard Developing Organizations (SDO) and open sources activities. Other group (SG11) is discussing SDN signaling. For its part, Internet Research Task Force (IRTF) has created the Software-Defined Networking Research Group (SDNRG) and Network Function Virtualization Research Group (NFVRG), which analyze the approaches that can be used in both technologies. Moreover, ETSI-ISG has delivered some initial requirements, service models and use cases for Network Function Virtualization.

We have presented the benefits of SDN/NFV in mobile networks. Based on the premises of both technologies, a whole overview of a possible framework is shown in Figure 3.

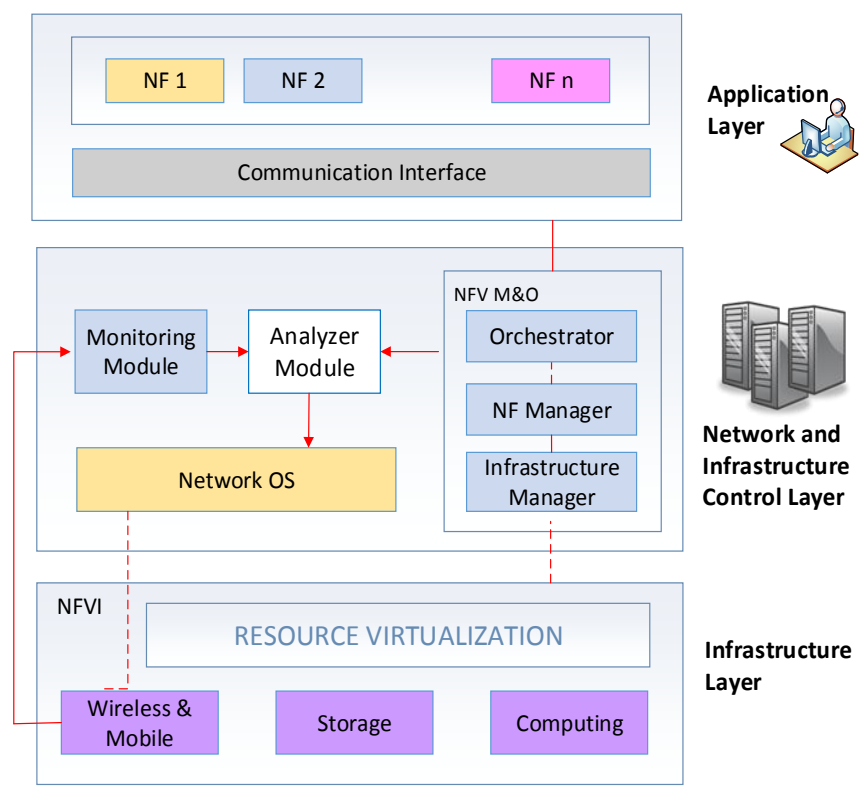

Fig. 3. SDN/NFV Architecture.
This architecture takes into account SDN and NFV technologies. On one hand, the framework presents a layered structure: data, control and application layers, in the same way that SDN architecture. Moreover, it takes advantage of NFV concept to allow the easy implementation and management of network functions, without the need to increase the hardware devices.

In the data layer, we have the current mobile infrastructure of the network operator providing support to a wide range of wireless and cellular technologies like WiFi, LTE, UTMS, GSM, among others. On top of this hardware layer, there is a virtualization layer to enable the virtualization of hardware devices. The resources could be in different locations and data centers and takes into account three components:

- Networking: These devices incorporate mobile technologies and OpenFlow protocol.

- Storage: This element can include Object storage or block storage (Swift and Cinder OpenStack) or another novel techniques.

- Computing: It include high volume servers. It could also use Openstack Nova.

The control layer is in charge of monitoring, analysis, management and orchestration of devices. Consist of four modules: monitoring, analyzer, network OS and NFV M\&O.

- Monitoring Module: This module is able to provide the complete low-level overview of the managed systems by mean of gathering metrics coming from different network devices.

- Analyzer Module: This module could give a deep analysis of the data in order to determine the suited behavior of the network. This module also can infer the recommended behavior of the network. The techniques used in the analysis can include: data mining, learning algorithms, pattern recognition, among others.

- Network OS: This module control de basic functions of the control layer. Also, it uses the OpenFlow or similar protocols to send instruction to the Infrastructure Layer elements. Its functionality is similar with an Operating Systems OS in computing.

- NFV M\&O: This module determines and organizes the actions to be executed in the system, the orchestration, the management of the resources and the control functions.

On the top of the architecture is located the application layer, which consists of two basic modules:

- Communication Interface: This module enables an open API to programmers to facilitate the development of new services.

- Network Functions: This module presents an scalable structure to create customized network functions or control applications.

This architecture enables users and developers a global view of IT infrastructure. Furthermore, the elements located on 
SDN/NFV control layer can adapt the network resources depending on the actual situation of the network and dynamically respond to failures or degradation of network performance.

\section{CONCLUSION}

The integration of digital services distributed over multiple mobile devices (laptop, tablet, cell phone, IoT) sharing high amounts of data (VoIP, streaming, digital images, e-gaming) have been limited by the closed-access and rigidity of actual IT infrastructure. Software Defined Networking and Network Function Virtualization have emerged as a part of the solution for the openness of the infrastructure and enabling to network administrator the dynamically customization of the network behavior.

This work presents a whole overview of the limitations of current IT infrastructure and introduces the novel concepts of SDN and NFV. Similarly, we describe the recent projects based on the integration of SDN/NFV with mobile infrastructure. The current trends and challenges in order to implement these advances in production networks are analyzed. Finally, we present an SDN/NFV architecture that integrates mobile and wireless technologies. It is clear that these paradigms bring new opportunities and create new business models for users, operators and service providers. However, it is fundamental the coordination between research community, industry and service operators in order to implement these advances in production networks.

\section{ACKNOWLEDGMENT}

The research leading to these results has been partially funded by the European Union's H2020 Program under the project SELFNET (671672). Ángel Leonardo Valdivieso Caraguay and Lorena Isabel Barona López are supported by the Secretaría Nacional de Educación Superior, Ciencia, Tecnología e Innovación SENESCYT (Quito, Ecuador) under Convocatoria Abierta 2012 and 2013 Scholarship Program. This work was partially supported by the "Programa de Financiación de Grupos de Investigación UCM validados de la Universidad Complutense de Madrid - Banco Santander".

\section{REFERENCES}

[1] B. Naudts, M. Kind, F. Westphal, S. Verbrugge, D. Colle, M. Pickavet. "Techno-economic Analysis of Software Defined Networking as Architecture for the Virtualization of a Mobile Network," in Proceedings of the European Workshop on Software Defined Networking, EWSDN, Darmstadt, Germany, October 2012, pp. 67-72.

[2] J. Q. Wang, F. Haijing, C. Chang. "Software Defined Networking for Telecom Operators: Architecture and Applications," in Proceedings of the 8th International Conference on Communications and Networking in China, CHINACOM, Guilin, China, August 2013, pp. 828-833.

[3] B. Haberland, F. Derakhshan, H. Grob-Lipski, R. Klotsche, W. Rehm, P. Schefczik, M. Soellner. "Radio Base Stations in the Cloud," in Bell Labs Technical Journal, vol. 18, no. 1, June 2013, pp 129-152.

[4] J. Costa-Requena. "SDN Integration in LTE Mobile Backhaul Networks," in Proceedings of the International Conference on Information Networking, ICOIN, Phuket, Thailand, February 2014, pp. 264-269.

[5] K. K. Yap, M. Kobayashi, R. Sherwood, T. Y. Huang, M. Chan, N Handigol, N. McKeown. "OpenRoads: Empowering Research in Mobile
Networks," in ACM SIGCOMM Computer Communication Review, vol. 40, no. 1, January 2010, pp. 125-126.

[6] X. Jin, L. E. Li, L. Vanbever, J. Rexford. "Softcell: Scalable and Flexible Cellular Core Network Architecture," in Proceedings of the Ninth ACM Conference on Emerging Networking Experiments and Technologies, ACM, California, CA, USA, December 2013, pp. 163174.

[7] TNOVA EU project, http://www.t-nova.eu/.

[8] UNIFY EU project, http://www.fp7-unify.eu/.

[9] A. L. Valdivieso Caraguay, L. I. Barona López, L. J. García Villalba. "Evolution and Challenges of Software Defined Networking," in Proceedings of the Workshop on Software Defined Networks for Future Networks and Services, Trento, Italy, November 2013, pp. 47-55.

[10] A. L. Valdivieso Caraguay, A. Benito Peral, L. I. Barona López, L. J. García Villalba. "SDN: Evolution and Opportunities in the Development IoT Applications," International Journal of Distributed Sensor Networks, IJDSN, May 2014, pp. 1-10.

[11] N. McKeown, T. Anderson, H. Balakrishnan, et al. "OpenFlow: Enabling Innovation in Campus Networks," in ACM SIGCOMM Computer Communication Review, vol. 38, no.2, April 2008, pp. 69-74.

[12] ETSI Industry Specification Group (ISG). "Network Function Virtualization (NFV) White Paper," in SDN and OpenFlow World Congress, Frankfurt, Germany, September 2013, pp. 1-16.

[13] H. Hawilo, A. Shami, M. Mirahmadi, R. Asal. "NFV: State of the Art, Challenges, and Implementation in Next Generation Mobile Networks (vEPC)," in IEEE Network, vol. 28, no. 6, November 2014, pp. 18-26.

[14] S. Costanzo, L. Galluccio, G. Morabito, S. Palazzo. "Software Defined Wireless Networks: Unbridling SDNs," in Proceedings of the European Workshop on Software Defined Networking, EWSDN, Darmstadt, Germany, October 2012, pp. 1-6.

[15] C. Chaudet, Y. Haddad. "Wireless Software Defined Networks: Challenges and Opportunities," in Proceedings of the 2013 IEEE International Conference on Microwaves, Communications, Antennas and Electronics Systems, COMCAS, Tel Aviv, Israel, October 2013, pp. $1-5$.

[16] K. K. Yap, R. Sherwood, M. Kobayashi, T. Y. Huang, M. Chan, N. Handigol, G. Parulkar. "Blueprint for Introducing Innovation into Wireless Mobile Networks," in Proceedings of the Second SIGCOMM Workshop on Virtualized Infrastructure Systems and Architectures, ACM, New Delhi, India, September 2010, pp. 25-32.

[17] A. Detti, C. Pisa, S. Salsano, N. Blefari-Melazzi. "Wireless Mesh Software Defined Networks (wmSDN)," in Proceedings of the $9^{\text {th }}$ IEEE International Conference on Wireless and Mobile Computing, Networking and Communications, WiMob, Lyon, France, October 2013, pp. 89-95.

[18] P. Dely, A. Kassler, N. Bayer. "Openflow for Wireless Mesh Networks," in Proceedings of the 20th IEEE International Conference on Computer Communications and Networks, ICCCN, Maui, Hawaii, July 2011, pp. 1-6.

[19] A. Basta, W. Kellerer, M. Hoffmann, K. Hoffmann, E.-D. Schmidt. "A Virtual SDN-Enabled LTE EPC Architecture: A Case Study for S-/PGateways Functions," in Proceedings of the Workshop on Software Defined Networks for Future Networks and Services, Trento, Italy, November 2013, pp. 8-14.

[20] S. B. H. Said, M. R. Sama, K. Guillouard, L. Suciu, G. Simon, X. Lagrange, J. M. Bonnin. "New Control Plane in 3GPP LTE/EPC Architecture for On-Demand Connectivity Service," in Proceedings of the Second IEEE International Conference on Cloud Networking, CloudNet, San Francisco, SF, USA, November 2013, pp. 205-209.

[21] K. Pentikousis, Y. Wang, W. Hu. "Mobileflow: Toward Softwaredefined Mobile Networks," in IEEE Communications Magazine, vol. 51, no. 7, July 2013, pp. 44-53.

[22] R. Riggio, T. Rasheed, F. Granelli. "EmPOWER: A Testbed for Network Function Virtualization Research and Experimentation," in Proceedings of the Workshop on Software Defined Networks for Future Networks and Services, Trento, Italy, November 2013, pp. 138-142. 
[23] R. Sherwood, G. Gibb, K. K. Yap, G. Appenzeller, M. Casado, N. McKeown, G. M. Parulkar. "Can the Production Network be the testbed?," in Proceedings of the $9^{\text {th }}$ USENIX Symposium on Operating Systems Design and Implementation, OSDI, Vancouver, BC, Canada, vol. 10, October 2010, pp. 365-378.

[24] A. Gudipati, D. Perry, L. E. Li, S. Katti. "SoftRAN: Software Defined Radio Access Network," in Proceedings of the Second ACM SIGCOMM Workshop on Hot Topics in Software Defined Networking, ACM, Hong Kong, China, August 2013, pp. 25-30.

[25] L. E. Li, Z. M. Mao, J. Rexford. "Toward Software-Defined Cellular Networks," in Proceedings of the European Workshop on Software Defined Networking, EWSDN, Darmstadt, Germany, October 2012, pp. 7-12.

[26] L. E. Li, Z. M. Mao, J. Rexford. "CellSDN: Software-Defined Cellular Networks," in Technical Report, Princeton University, 2012.

[27] ICT-CROWD EU project, http://www.ict-crowd.eu/.

[28] CITYFLOW project, http://www.onesource.pt/cityflow/site/.

[29] OFELIA EU project, http://www.fp7-ofelia.eu/.

[30] EUKOREA EU project, http://eukorea-fire.eu/pilots/.

[31] F. Schneider, T. Egawa, S. Schaller, S. I. Hayano, M. Schöller, F. Zdarsky. "Standardizations of SDN and Its Practical Implementation," in NEC Technical Journal, vol. 8, no. 2, April 2014, pp. 16-20. 